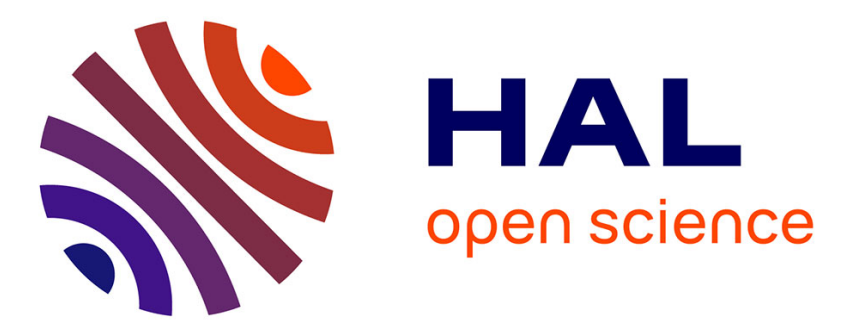

\title{
Bekki-Nozaki Amplitude Holes in Hydrothermal Nonlinear Waves
}

Javier Burguete, Hugues Chaté, François Daviaud, Nathalie Mukolobwiez

\section{To cite this version:}

Javier Burguete, Hugues Chaté, François Daviaud, Nathalie Mukolobwiez. Bekki-Nozaki Amplitude Holes in Hydrothermal Nonlinear Waves. Physical Review Letters, 1999, 82, pp.3252-3255. 10.1103/PhysRevLett.82.3252 . cea-01373884

\section{HAL Id: cea-01373884 https://hal-cea.archives-ouvertes.fr/cea-01373884}

Submitted on 29 Sep 2016

HAL is a multi-disciplinary open access archive for the deposit and dissemination of scientific research documents, whether they are published or not. The documents may come from teaching and research institutions in France or abroad, or from public or private research centers.
L'archive ouverte pluridisciplinaire $\mathbf{H A L}$, est destinée au dépôt et à la diffusion de documents scientifiques de niveau recherche, publiés ou non, émanant des établissements d'enseignement et de recherche français ou étrangers, des laboratoires publics ou privés. 


\title{
Bekki-Nozaki Amplitude Holes in Hydrothermal Nonlinear Waves
}

\author{
Javier Burguete, ${ }^{1,2}$ Hugues Chaté, ${ }^{1}$ François Daviaud, ${ }^{1}$ and Nathalie Mukolobwiez ${ }^{1}$ \\ ${ }^{1}$ CEA-Service de Physique de l'Etat Condensé, Centre d'Etudes de Saclay, 91191 Gif-sur-Yvette, France \\ ${ }^{2}$ Departamento de Física y Matemática Aplicada, Facultad de Ciencias, Universidad de Navarra, 31080 Pamplona, Spain
}

(Received 25 November 1998)

\begin{abstract}
We present and analyze experimental results on the dynamics of hydrothermal waves occurring in a laterally heated fluid layer. We argue that the large-scale modulations of the waves are governed by a one-dimensional complex Ginzburg-Landau equation (CGLE). We determine quantitatively all the coefficients of this amplitude equation using the localized amplitude holes observed in the experiment, which we show to be well described as Bekki-Nozaki hole solutions of the CGLE. [S0031-9007(99)08940-1]
\end{abstract}

PACS numbers: 47.20.Dr, 05.45.-a, 47.27.Te, 47.35.+i

The status and nature of the so-called amplitude equations which can be derived in the vicinity of symmetrybreaking instabilities is now well established $[1,2]$. They are "universal" insofar as they essentially depend on the symmetries of the physical system and of its bifurcated solutions, but also because they often remain valid, at least at a qualitative level, even far away from the instability threshold $[3,4]$. However, determining accurately the coefficients of the underlying relevant amplitude equation from experimental data remains a difficult task, especially in these far-from-threshold regimes.

The complex Ginzburg-Landau equation (CGLE), which describes the large-scale modulations of the bifurcated solutions near oscillatory instabilities, is perhaps the most-studied amplitude equation [1]. This privileged situation is due to both its relevance to many experimental situations and to the variety of its dynamical behavior, in particular, its spatiotemporal chaos regimes. One of the landmarks of the CGLE is that it possesses localized "defect" solutions. Even in one space dimension, where no topological constraint exists, numerical simulations of the CGLE [5,6] and analytical $[7,8]$ work have revealed the existence and importance of various amplitude hole solutions, which can often be seen as the "building blocks" of the complex spatiotemporal dynamics observed. In particular, the one-parameter family of traveling hole solutions discovered by Bekki and Nozaki [9] has been shown to play an important dynamical role in a large portion of parameter space including in regions where they are linearly unstable [5]. Similar objects have been identified in various experimental contexts of a priori relevance, e.g., Rayleigh-Bénard convection and coupled wakes [10]. However, to our knowledge, there is still no case where a direct comparison with known solutions of the CGLE could be achieved.

In this Letter, we present a quantitative comparison of localized amplitude holes observed in an experiment with hole solutions of the CGLE, the relevant amplitude equation. We use the observed holes to fully determine the coefficients of the underlying CGLE. This provides clear-cut evidence of Bekki-Nozaki holes in an experi- mental context. Our system is a long, straight, and narrow convection cell in which a thin fluid layer with a free surface is subjected to a horizontal temperature gradient. Hydrothermal nonlinear waves appear via a direct Hopf bifurcation, indicating the relevance of the CGLE. The spatiotemporal dynamics of the waves exhibits localized amplitude holes. The basic scales of the equivalent CGLE are determined using the regular part of the wave trains. Data collected in the vicinity of amplitude holes show that they have the structure of Bekki-Nozaki solutions. This also provides estimates of the remaining coefficients of the CGLE, an approach which, we argue, could be efficient in other experimental contexts. Finally, the overall consistency of our results is checked.

The experimental setup is schematically described in Fig. 1. A layer of fluid [silicon oil of viscosity $\nu=$ $0.65 \mathrm{cS}$ (centistoke) and Prandtl number $P=10$ ] of height $h$ is confined between two copper blocks maintained at fixed temperatures $T_{+}$and $T_{-}$by thermostated water circulation, and a bottom glass plate. This forms a straight, narrow channel of length $L_{x}=25 \mathrm{~cm}$ and width $L_{y}=2 \mathrm{~cm}$. As soon as the temperature difference $\Delta T=T_{+}-T_{-}$is not zero, a basic flow sets in. It consists of a surface flow towards the cold side with a bottom recirculation. Increasing $\Delta T$, the basic flow becomes unstable to traveling hydrothermal waves [11] via a supercritical Hopf bifurcation [12]. We observe these waves by low-contrast shadowgraphy, which captures the vertical average of the temperature

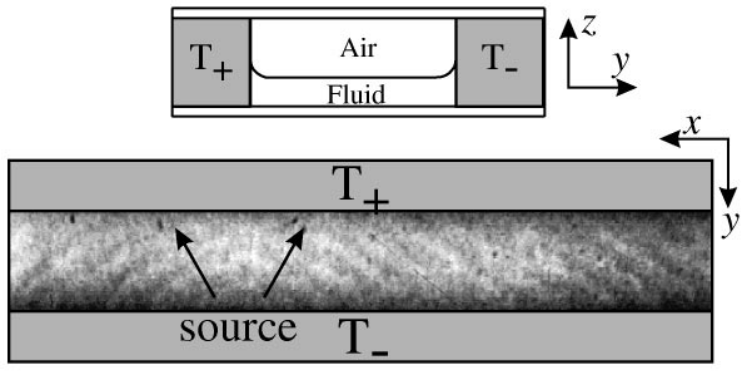

FIG. 1. Experimental cell and basic wave pattern. Above: Schematic side view. Below: Top view and instantaneous shadowgraphic image of the wave pattern. 
gradient variations (surface waves exist, but their effect is negligible). In this geometry, the waves propagate away from a "source region" located arbitrarily on the cold wall, and the end boundaries at $x=0, L_{x}$ act as sinks with no apparent reflection (Fig. 1). For $h=1.2 \mathrm{~mm}$, corresponding to the experiment reported below, the source region emits curved waves which become planar further away (Fig. 1, bottom) and propagate along the $x$ axis.

Figure 2a presents a typical spatiotemporal evolution as obtained from the acquisition, with a fixed-gain camera, of a single 512-pixel line (of negligible width) along the $x$ axis in the center of the cell. Here, the source appears as a rather ill-defined, erratic object. (Closer to the Hopf bifurcation, steady, regular, evolution is observed.) Fourier analysis of diagrams such as Fig. 2a reveals that on each side of the source only waves propagating away from the source are present and that they are approximately monochromatic (the second harmonic is 2 orders of magnitude smaller). More precisely, restricting ourselves to one side of the source (say $x \geq 90$ on Fig. 2a), we can write the recorded physical variable:

$$
V(x, t)=A(x, t) \exp \left[i\left(k_{0} x-\omega_{0} t\right)\right]+\text { c.c. },
$$

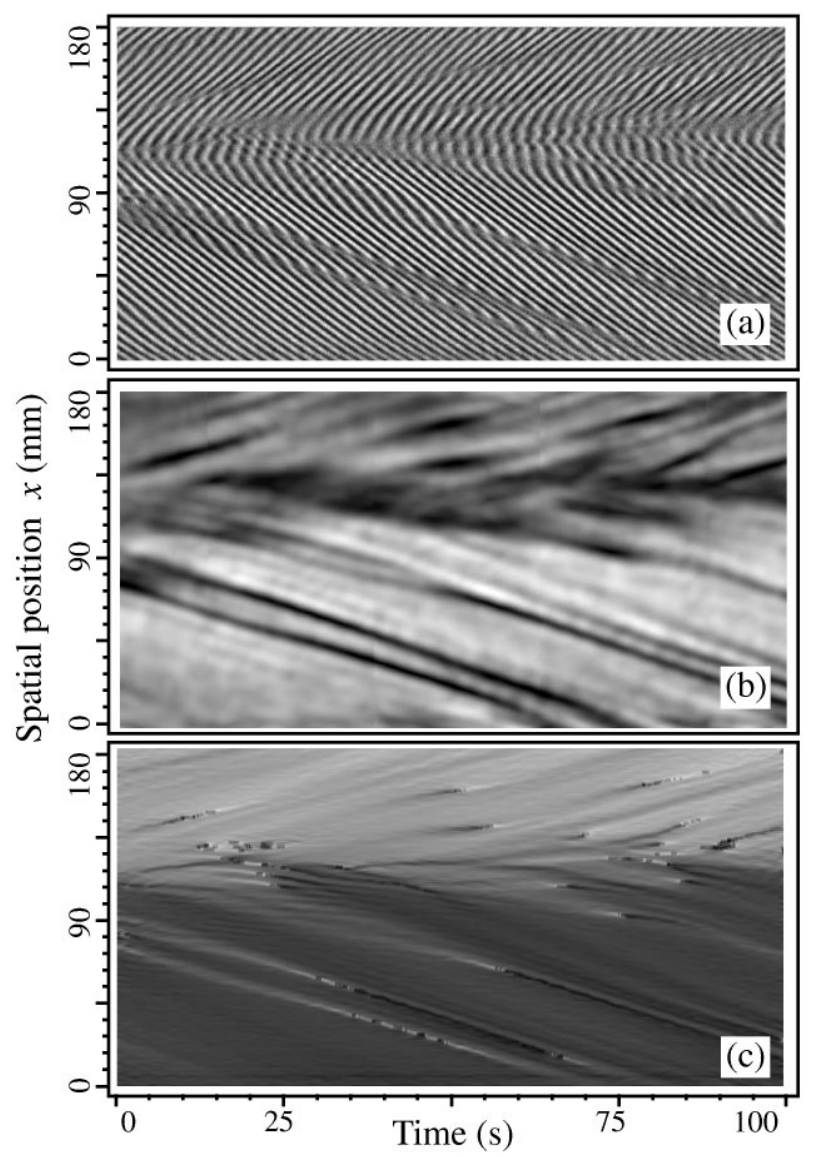

FIG. 2. Spatiotemporal evolution of the wave pattern at $h=$ $1.2 \mathrm{~mm}$ and $\Delta T=5.1 \mathrm{~K}$ (yielding a Marangoni number $\mathrm{Ma} \simeq$ 950). Only a central portion of length $18.5 \mathrm{~cm}$ is shown during 100 s. (a) Original data; (b) evolution of the modulus $|A|$ (black: $|A|=0$; white: $|A|=1$ ); and (c) phase gradient $k$ (dark: $k<0$; bright: $k>0$ ). where $k_{0}$ is the dominant wavelength, $\omega_{0}$ is the basic frequency, and $A$ is a one-dimensional complex field describing the (large-scale) modulations of this wave. (On the other side of the source, one has to change the sign of $k_{0}$.)

Using complex demodulation techniques, $A$ can be extracted from the experimental data. Figures $2 \mathrm{~b}$ and $2 \mathrm{c}$ show the space-time evolution of $|A|$ and $k=q \pm k_{0}$, where $q=\partial_{x} \arg (A)$. In these pictures, the localized deformations of the waves visible in Fig. 2a clearly appear as propagating amplitude holes across which the phase gradient varies rapidly. At some space-time points, $|A|$ even vanishes and the phase gradient diverges: A spacetime dislocation occurs (Fig. 2a). The amplitude holes can be seen as the objects mediating the evolution to wave patterns more regular than those emitted by the source.

Our system clearly calls for a one-dimensional model. The waves arise via a supercritical Hopf bifurcation. Away from the source, they propagate only in one direction. All of this indicates that the evolution of $A$ could be governed by a single CGLE on each side of the source, even though the regime studied here takes place at a finite distance from threshold (for $h=1.2 \mathrm{~mm}, \Delta T_{\mathrm{c}} \simeq 4.3 \mathrm{~K}$, and thus the relative distance to threshold is $\varepsilon=0.19$ for $\Delta T=5.1 \mathrm{~K})$. We thus suppose that $A$ obeys

$$
\begin{aligned}
\tau_{0}\left(\partial_{t}-v_{\mathrm{g}} \partial_{x}\right) A= & \varepsilon A+\xi_{0}^{2}(1+i \alpha) \partial_{x x} A \\
& -g(1+i \beta)|A|^{2} A,
\end{aligned}
$$

where $v_{\mathrm{g}}$ is the group velocity of the waves, $\tau_{0}$ and $\xi_{0}$ are the basic time and length scales of the wave modulations, and $g$ is a real number. Below, we estimate, from the data of Fig. 2, all of the coefficients of Eq. (2) and check the overall consistency of our hypothesis.

The linear part of the variation of the local frequency $\omega$ with the local wave number $k$ yields our estimate of the group velocity: $v_{\mathrm{g}}=\partial \omega / \partial k \simeq-1.16 \mathrm{~mm} / \mathrm{s}$ (Fig. 3a). This is consistent with the average value of the velocity of small perturbations, estimated at $-1.15 \pm 0.25 \mathrm{~mm} / \mathrm{s}$, to be compared to the phase velocity $v_{\phi}=\omega_{0} / k_{0} \simeq$ $-2.8 \mathrm{~mm} / \mathrm{s}$. This confirms that the source is indeed a source, since perturbations do propagate outward.

Figure $3 \mathrm{~b}$ shows the variation of $|A|$ with $k$ as determined from the portion of Fig. 2 at the left of the source $(x \leq 90 \mathrm{~mm})$. The maximum amplitude is observed for the basic wave number: $k_{0} \simeq-1.11 \mathrm{~mm}^{-1}$. Space-time points away from the localized amplitude holes correspond to the large $|A|$ (say, $|A|>0.5$ ) portion of the curve. Locally, around these points, the solution of (2) is expected to be close to one of the phase-winding solutions of wave vector $q=k+k_{0}$ (see, e.g., [1]):

$$
\begin{array}{r}
A=A_{q} \exp \left[i\left(q x-\omega_{q} t\right)\right] \quad \text { with } A_{q}^{2}=\left(\varepsilon-\xi_{0}^{2} q^{2}\right) / g \\
\quad \text { and } \omega_{q}=\left[\varepsilon \beta+(\alpha-\beta) \xi_{0}^{2} q^{2}\right] / \tau_{0}-v_{\mathrm{g}} q .
\end{array}
$$

The linear variation of $|A|^{2}$ with $q^{2}$ is confirmed in Fig. 3c, yielding $\xi_{0} / \sqrt{\varepsilon} \simeq 2.53 \mathrm{~mm}$ and $\sqrt{\varepsilon / g} \simeq$ 0.00054 (a.u.). Note that we thus have $L_{x} \gg \xi_{0} / \sqrt{\varepsilon} \approx$ 
(a)

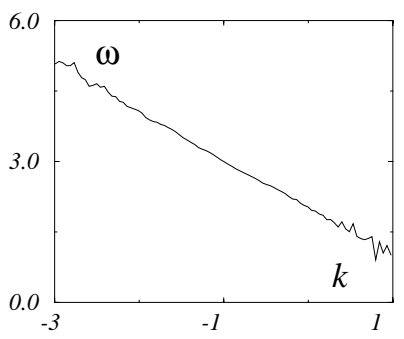

(c)

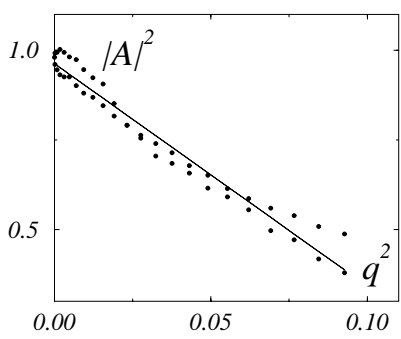

(b)

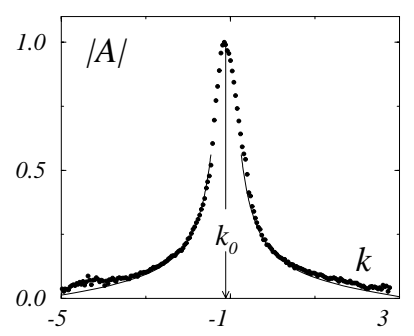

(d)

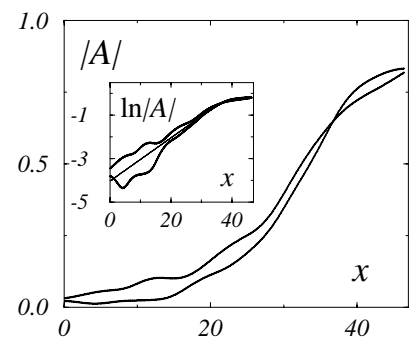

FIG. 3. Analysis of the data of Fig. 2 situated at the left of the source $(x \leq 90 \mathrm{~mm})$. (a)-(c) For each bin around a given $k$ value, all the corresponding space-time points were determined, and averages calculated on each bin. (a) Local frequency $\omega$ vs local wave number $k$; the slope of a linear fit gives the group velocity $v_{\mathrm{g}}$. (b) Local amplitude $|A|$ vs $k$; solid line: see text. (c) $|A|^{2}$ vs $q^{2}$ [same data as in (b)]. (d) Two instantaneous profiles of $|A|(x)$ near the source taken at time 0 and $30 \mathrm{~s}$; inset: $\ln |A|$ vs $x$ and linear fit (thin line).

$3 / k_{0}$ : The cell is effectively "infinite" and the variations of $A$ occur on scales significantly larger than the basic length $k_{0}^{-1}$.

Time scale $\tau_{0}$ can be estimated from the real part of the spatial linear growth rate of waves near the source, which is equal to $\varepsilon / \tau_{0} v_{\mathrm{g}}$ [4]. From Fig. $3 \mathrm{~d}$, we find $\tau_{0} / \varepsilon \simeq 8.5 \pm$ $0.5 \mathrm{~s}$, about 4 times the basic period $2 \pi / \omega_{0} \simeq 2.03 \mathrm{~s}$, confirming that the variations of $A$ are slow compared to the basic oscillations. Note that $\tau_{0}$ is of the same order as the viscous diffusion time $h^{2} / \nu=2.2 \mathrm{~s}$.

At this stage, all of the basic scales of Eq. (2) have been estimated. To determine the remaining two parameters $\alpha$ and $\beta$, global quantities deriving from the "wave part" of the data could, in principle, be sufficient. For example, Fig. 3a could be used to extract the expected variation of $\omega_{q}$ with $q$. But the data is too noisy to yield any meaningful estimate of $\alpha$ and $\beta$. Moreover, as long as the source is not controlled, the "input" waves cannot be varied at will to explore the family of solutions (3), contrary to other experimental situations [4]. We now focus instead on the localized amplitude holes already mentioned.

Many localized, propagating objects connecting two phase-winding solutions have been observed numerically in the one-dimensional CGLE [5]. Analytical methods are largely limited, so far, to solutions depending only on the reduced variable $\xi=x-v_{\mathrm{h}} t$, where $v_{\mathrm{h}}$ is the (constant) velocity of the object $[7,8]$. Using this ansatz, the CGLE reduces to a third-order ordinary differential equation

(ODE) whose fixed points are the phase-winding solutions (3). Localized objects connecting two such solutions of wave vector $q_{\mathrm{L}}$ and $q_{\mathrm{R}}$ appear, within the ansatz, as homoclinic $\left(q_{\mathrm{L}}=q_{\mathrm{R}}\right)$ or heteroclinic $\left(q_{\mathrm{L}} \neq q_{\mathrm{R}}\right)$ orbits.

The holes observed in Fig. 2 are not stable structures connecting two infinite phase-winding solutions, but they subsist long enough, and can be sufficiently isolated to reveal that their wings are indeed well described by phasewinding solutions. (As a matter of fact, we repeatedly assimilated, above, the large-amplitude regions separating the holes to portions of these solutions.) Figure 4 shows an isolated hole extracted from Fig. 2. One can measure rather accurately the two wave numbers $q_{\mathrm{L}}=0.13$ and $q_{\mathrm{R}}=-0.32$ (in the CGLE frame) connected by the central hole, which can thus be tentatively seen as a heteroclinic orbit in the ODE ansatz.

All sufficiently localized structures on Fig. 2 also connect two different wave numbers. This rules out the homoclinic holes recently studied by van Hecke [6], and leave, as possible candidates, the family of hole solutions found by Bekki and Nozaki [9]. The explicit form of these solutions is too lengthy to be given here (see, e.g., [8]). They form a one-parameter family (at fixed $\alpha$, $\beta$ ) which can be parametrized by, e.g., the velocity $v_{\mathrm{h}}$ of the hole. They take the shape of an exponentially localized amplitude hole with a minimum amplitude $|A|_{\min }$ accompanied by a rapid phase shift $\sigma$.

To compare data such as that of Fig. 4 to these solutions, we need to determine the values of $\alpha$ and $\beta$ and the "optimal" solution of the corresponding family. We proceed as follows: We estimate $q_{\mathrm{L}}, q_{\mathrm{R}}$, and $|A|_{\text {min }}$ from the data since we found these were the characteristics of the hole for which the most accurate measurement can be made. We find, for all values of the $(\alpha, \beta)$ plane where it exists, the Bekki-Nozaki hole solution with the measured value of $q_{\mathrm{L}}+q_{\mathrm{R}}$. We then select the (codimension 1) subsets of the $(\alpha, \beta)$ plane where, moreover, this hole solution possesses the measured value of $q_{\mathrm{L}}$ or the estimated value of $|A|_{\text {min }}$ (Fig. 5, dashed lines). These two lines intersect, yielding the desired values of $\alpha$ and $\beta$. Taking into account the error bars on $q_{\mathrm{L}}, q_{\mathrm{R}}$, and $|A|_{\text {min }}$, we find $\alpha=-1.5 \pm 0.5$ and $\beta=-0.4 \pm 0.05$. By the

(a)

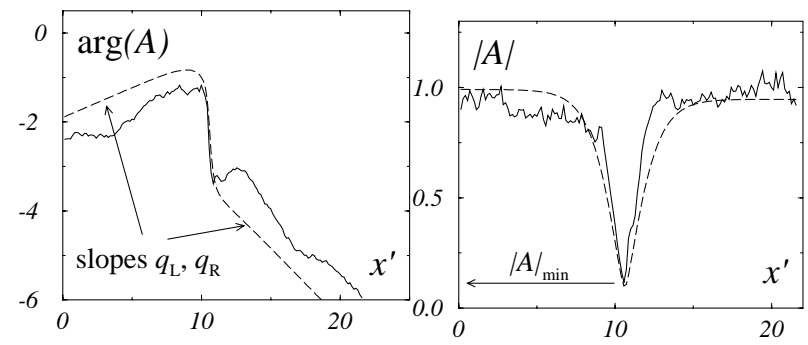

FIG. 4. Amplitude hole. Solid lines: Experimental data extracted from a cut of Fig. 2 at $t=60 \mathrm{~s}$. Dashed lines: Corresponding Bekki-Nozaki hole solution of the equivalent CGLE (rescaled variable $x^{\prime}=x \sqrt{\varepsilon} / \xi_{0}$ ). 


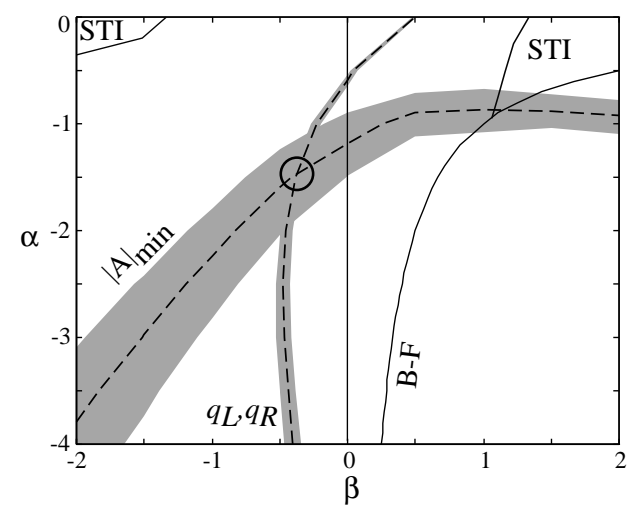

FIG. 5. Parameter plane of the CGLE. STI: spatiotemporal intermittency regions (from [5]). B-F: Benjamin-Feir line below which all solutions (3) are unstable. Dashed lines: see text (grey areas: error bars). Circle: estimated $(\alpha, \beta)$ values.

same token, the hole solution is also uniquely determined (Fig. 4, dashed lines). Its velocity $v_{\mathrm{h}}$, its width, and its phase shift $\sigma$ are all found consistent with the data.

We repeated the procedure for other amplitude holes found in Fig. 2. We found almost the same $\alpha$ and $\beta$ values, to the accuracy of the estimates. This strengthens the confidence in the results, since different objects moving at different velocities, connecting different wave numbers all yield the same parameter values. We also performed a final check, by plotting, for the estimated values of $\alpha$ and $\beta$, the variation of $|A|_{\min }$ with the local phase gradient at the "bottom" of the hole along the family of solutions (Fig. 3b, solid line). The agreement with the small- $|A|$ values measured on Fig. 2 is very good. This is an additional indication that all of the low-amplitude points are indeed located "inside" Bekki-Nozaki holes.

For the estimated values of $\alpha$ and $\beta$, the CGLE is in the parameter region where the phase-winding solutions (3) are linearly stable for $|q|$ small enough, and no sustained spatiotemporal disorder exists in one space dimension [5]. Moreover, the Bekki-Nozaki amplitude holes are linearly unstable [13]. This is not in contradiction with the dynamics observed in the experiment: the BekkiNozaki holes, although unstable, exist, and can constitute important building blocks of even chaotic dynamics [5]. The waves emitted by the source can be locally attracted to this family of unstable fixed points before escaping along its unstable manifold (a mechanism also invoked by van Hecke in [6]). The tendency of the waves trains to become more regular away from the source (see Fig. 2) is consistent with disorder being only transient in the CGLE with the estimated parameter values.

In summary, we have presented experimental results on the dynamics of the nonlinear hydrothermal waves traveling in a laterally heated fluid layer. We have shown that, although the regime studied here is rather far from the onset of waves, the large-scale modulations of the basic pattern are governed by a one-dimensional complex Ginzburg-Landau equation and we estimated its full set of coefficients. This was made possible by showing that the localized amplitude holes observed experimentally correspond to the Bekki-Nozaki hole solutions of the CGLE. The overall consistency of our results was checked. Since the operating regime of the CGLE at the estimated parameter values does not exhibit sustained disorder, it would be interesting to analyze experimental data collected at other parameter values in the hope of reaching spatiotemporal chaos regimes of the type exhibited by the CGLE. This is left for future work, together with an attempt to obtain a better control of the system by forcing the behavior of the source. More generally, we believe that using the localized structures or defects of patternforming systems to determine quantitatively their relevant amplitude equations can be a rewarding approach to this difficult experimental problem.

J.B. thanks the Spanish government for support through Project No. PB95-0578A (DGICYT) and a postdoctoral grant (SEUI, Ministerio de Educación y Ciencia).

[1] M. C. Cross and P.C. Hohenberg, Rev. Mod. Phys. 65, 851 (1993); Y. Kuramoto, Chemical Oscillations, Waves, and Turbulence (Springer-Verlag, Berlin, 1984).

[2] P. Manneville, Dissipative Structures and Weak Turbulence (Academic Press, Boston, 1990); S. Sasa, Physica (Amsterdam) 108D, 45 (1997).

[3] T. Leweke and M. Provansal, J. Fluid. Mech. 288, 265 (1995); P. Kolodner, S. Slimani, N. Aubry, and R. Lima, Physica (Amsterdam) 85D, 165 (1995); S. Jarre, P. Le Gal, and M.P. Chauve, Phys. Fluids 8, 2985 (1996); Y. Liu and R. E. Ecke, Phys. Rev. Lett. 78, 4391 (1997).

[4] V. Croquette and H. Williams, Physica (Amsterdam) 37D, 300 (1989); Phys. Rev. A 39, 2765 (1989).

[5] H. Chaté, Nonlinearity 7, 185 (1994).

[6] M. van Hecke, Phys. Rev. Lett. 80, 1896 (1998).

[7] W. van Saarloos and P.C. Hohenberg, Physica (Amsterdam) 56D, 303 (1992); Physica (Amsterdam) 69D, 209(E) (1993).

[8] R. Conte and M. Musette, Physica (Amsterdam) 69D, 1 (1993).

[9] N. Bekki and K. Nozaki, Phys. Lett. 110A, 133 (1985).

[10] J. Lega, B. Janiaud, S. Jucquois, and V. Croquette, Phys. Rev. A 45, 5596 (1992); J. M. Flesselles, V. Croquette, and S. Jucquois, Phys. Rev. Lett. 72, 2871 (1994); P. Le Gal, M. P. Chauve, I. Peschard, and S. Jarre, Curr. Top. Phys. Fluids 1, 307 (1994).

[11] M. K. Smith and S.H. Davis, J. Fluid Mech. 132, 119 (1983); J.-F. Mercier and C. Normand, Phys. Fluids 8, 1433 (1996); F. Daviaud and J. M. Vince, Phys. Rev. E 48, 4432 (1993); D. Schwabe, U. Möller, J. Schneider, and A. Scharmann, Phys. Fluids 4, 2368 (1992).

[12] N. Mukolobwiez, A. Chiffaudel, and F. Daviaud, Phys. Rev. Lett. 80, 4661 (1998).

[13] H. Sakaguchi, Prog. Theor. Phys. 85, 417 (1991); H. Chaté and P. Manneville, Phys. Lett. A 171, 183 (1992); S. Sasa and T. Iwamoto, Phys. Lett. A 175, 289 (1993); S. Popp, O. Stiller, I. S. Aranson, and L. Kramer, Physica (Amsterdam) 84D, 398 (1995). 